\title{
Passing Beyond a Wing and a Prayer after Hospital Discharge
}

\author{
Luke O. Hansen, MD MHS \\ Department of Medicine, Division of Hospital Medicine, Northwestern University Feinberg School of Medicine, Chicago, IL, USA.
}

J Gen Intern Med 30(4):390-1

DOI: $10.1007 / \mathrm{s} 11606-015-3186-9$

(c) Society of General Internal Medicine 2015

$\mathrm{T}$ here is an anxious moment in American football, when a trailing team with seconds left in the game may attempt to win by throwing a long pass down the field, hoping for a lastsecond touchdown. As a spectator, it is thrilling but frustrating - if only the trailing team had worked better together, had been a better team, this high-risk and low-yield strategy might have been avoided. Such a gambit has been termed the "Hail Mary" pass, and the analogy can be made between it and the often disappointing approach to handoffs of care following hospitalization. In recent years, with impetus for change provided by the Hospital Readmission Reduction Penalty within the Affordable Care Act, as well as interest across diverse payors to reward value in healthcare, the standard of care at hospital discharge is changing. Post-discharge callbacks, pharmacist-guided medication reconciliation, and timely ambulatory follow-up after discharge are examples of tactics that are proliferating in an attempt to provide post-discharge care that can guarantee wellness following hospitalization. And while there is evidence of improvement in rates of rehospitalization, ${ }^{1}$ the evidence is also mixed, with many of these pillars of high-quality care transitions failing to reliably demonstrate effectiveness. $^{2-4}$ Despite intense research attention on care transitions in recent years, our understanding of readmission risk and how to remedy risk with interventions generalizable across socioeconomic spectra and care settings is remarkably limited. Is poor teamwork across the discharge transition limiting the effectiveness of our interventions and continuing to leave only "Hail Mary" options?

In this issue of JGIM, Jones and colleagues describe the key roles interprofessional relationships and communication play in establishing the climate for success of transitional care management. ${ }^{5}$ Their portrait of a care period characterized by unclear provider accountability and inadequate knowledge on the part of both providers and patients is painfully familiar to those who treat patients across the post-hospital care transition. ${ }^{6}$ Their findings are disquieting but not altogether surprising: an implicit lack of investment in the transitional care of the discharged patient that providers attribute to inadequate time, failures of communication attributed to poor

Published online February 4, 2015 technological resources in systems that continue to depend largely on one-way pager and fax technology, and persistent clinical loose ends despite proliferation of health information technology.

This study reinforces and adds qualitative physician perspective to previous findings regarding poor discharge handoffs. It has been reported that of the $25 \%$ of discharged patients requiring additional workup, over one-third fail to complete recommended follow-up. ${ }^{7}$ Direct communication is the exception rather than the norm, and written communication via discharge summary has occurred at the time of the first post-discharge visit less than one-third of the time. ${ }^{8}$ The standard of care for discharge summary completion remains stubbornly stuck at thirty days in many hospitals.

Several areas of focus are appropriate for further inquiry. Jones et al. have documented provider narrative regarding weaknesses of care and communication. Additional quantitative work remains to further define how frontline providers should relate to each other as they implement best practices in order to maximize the safety of hospital care transitions. Jones and colleagues suggest that shortcomings in communication may be influenced by poor relationships between inpatient and outpatient providers. Literature looking specifically at how poor teamwork between inpatient and outpatient providers occurs and what characterizes high functioning transitional care teams is lacking.

The authors' data indicate that relational continuity among providers could be protective and strengthen handoffs. Previous work has indicated that continuity between patients and providers appears protective after hospitalization, ${ }^{9}$ but additional studies exploring how to support more stable hospitalist/ outpatient generalist dyads or perhaps even revival of a more traditional generalist model - the "comprehensive care physician" 10 - seem indicated. Transitional care models assigning nurse or social work clinicians to support care transitions longitudinally across care settings may derive some benefit from the provision of an otherwise absent relationship bridge across the care transition. ${ }^{11,12}$

Providers that were interviewed were quick to attribute poor communication to the time necessary for what have been referred to as "warm handoffs" - patient handoffs between providers that involve conversation and dynamic inquiry regarding care plans, rather than static data transmission. Additional research on more robust relational continuity and communication intensity must consider opportunity costs and include careful assessment of balancing metrics, including 
whether more time-intensive handoffs might lead to reductions in the time invested in other essential discharge activities such as medication reconciliation. This balance of cost and benefit cannot be determined without improved understanding of clinical and social risks for rehospitalization. With wide variation in both risk and the financial and time cost of interventions, additional research to understand biopsychosocial risk for poor outcomes following rehospitalization continues to be needed to assign high intensity care transition approaches to appropriate risk cohorts.

Finally, it is appropriate to consider the patient's role as a team member supporting high quality discharge. While Jones and colleagues have characterized providers' experiences, their portrait of provider relationships and communication calls for the addition of the patient voice in a qualitative fashion. How would patients and their caregivers characterize their role in the handoff? We often picture them as passive, but in fact it may be best for their health if they share the work of high-quality care transitions. There is emerging literature on the importance of patient activation, ${ }^{13}$ and a relationship between this characteristic and successful transitions seems likely, but is in need of further description. Further qualitative work could be highly informative, regarding patient experience with care transitions and roles patients may be drawn to as members of the care transitions team, rather than as passive vehicles.

Desperate football teams fall back on the Hail Mary Pass as their last resort, often after a series of mistakes throughout the game, sometimes because they do not field the right team members, and always because they badly want to win. Our healthcare teams similarly want very much, often desperately, to do the job right. But can we say we are diligent about building interprofessional relationships and supporting them with active communication? Or that we adequately consider colleagues and patients as essential teammates in our work? Jones and colleagues are reminding us that success may not only depend on high quality process, but also may require longitudinal team-building among caregivers to turn our work into dependable wins.

Corresponding Author: Luke O. Hansen, MD MHS; Department of Medicine, Division of Hospital MedicineNorthwestern University Feinberg School of Medicine, 211 East Ontario Street, Suite 700, Chicago, IL 60610, USA (e-mail: l-hansen@northwestern.edu).

\section{REFERENCES}

1. Gerhardt G, Yemane A, Hickman P, Oelschlaeger A, Rollins E, Brennan N. Medicare readmission rates showed meaningful decline in 2012. Med Medicaid Res Rev. 2013;3(2).

2. Field T, Ogarek J, Garber L, Reed G, Gurwitz J. Association of early postdischarge follow-up by a primary care physician and 30-day rehospitalization among older adults. J Gen Intern Med. 2014;2014:1-7.

3. Kwan JL, Lo L, Sampson M, Shojania KG. Medication reconciliation during transitions of care as a patient safety strategy a systematic review. Ann Intern Med. 2013;158(5_Part_2):397-403.

4. Mistiaen P, Poot E. Telephone follow-up, initiated by a hospital-based health professional, for postdischarge problems in patients discharged from hospital to home. Cochrane Database Syst Rev. 2006;4.

5. Jones CD. ([SPI \#3056]). A failure to communicate: a qualitative exploration of care coordination between hospitalists and primary care providers around hospitalizations. J Gen Intern Med.

6. Krumholz HM. Post-hospital syndrome - an acquired, transient condition of generalized risk. N Engl J Med. 2013;368(2): 100-2.

7. Moore C, McGinn T, Halm E. Tying up loose ends: discharging patients with unresolved medical issues. Arch Intern Med. 2007;167(12):1305-11.

8. Kripalani S, LeFevre F, Phillips CO, Williams MV, Basaviah P, Baker DW. Deficits in communication and information transfer between hospitalbased and primary care physicians. JAMA. 2007;297(8):831-41.

9. van Walraven C, Mamdani M, Fang J, Austin PC. Continuity of care and patient outcomes after hospital discharge. J Gen Intern Med. 2004;19(6):624-31.

10. Meltzer DO, Ruhnke GW. Redesigning care for patients at increased hospitalization risk: the comprehensive care physician model. Health Aff. 2014;33(5):770-7.

11. Coleman EA, Smith JD, Frank JC, Min SJ, Parry C, Kramer AM. Preparing patients and caregivers to participate in care delivered across settings: the Care Transitions Intervention. J Am Geriatr Soc. 2004;52(11): 1817-25.

12. Naylor M, Brooten D, Campbell R, et al. Comprehensive discharge planning and home follow-up of hospitalized elders: a randomized clinical trial. JAMA. 1999;281(7):613.

13. Hibbard JH, Greene J. What the evidence shows about patient activation: better health outcomes and care experiences; fewer data on costs. Health Aff. 2013;32(2):207-14. 